\title{
Dermoscopic features of acral melanocytic nevi in a case series from Mexico*
}

\author{
Roger Adrián González-Ramírez ${ }^{1,2}$, Carolina Guerra-Segovia3, Verónica Garza-Rodríguez ${ }^{3}$ \\ Pamela Garza-Báez ${ }^{3}$, Minerva Gómez-Flores ${ }^{3}$,Jorge Ocampo-Candiani ${ }^{3}$
}

DOI: http:/ / dx.doi.org/10.1590/abd1806-4841.20186695

\begin{abstract}
BACKGROUnD: Pigmented lesions on acral sites are common; clinical differentiation of nevi and early melanoma can be challenging. In these cases, dermoscopy can provide a more accurate diagnosis. Most dermoscopic patterns on acral skin have been described in Asian and European populations, while there are few studies in Latin American populations.

ОвлестіVEs: To determine the frequency of pigmented lesions in volar skin and their dermoscopic patterns in a Mexican population. Methods: An observational, descriptive, cross-sectional study was performed in Hispanic patients with the presence of at least one pigmented lesion on acral skin. Clinical and dermoscopic images were obtained. These were subsequently evaluated independently by two dermatologists trained and experienced in dermoscopy.

RESULTS: A total of 582 pigmented lesions on volar skin were diagnosed in 321 patients. Overall, prevalence of acral pigmented lesions on volar skin was $6.8 \%$. For both observers, parallel furrows were the most frequent pattern described, but for observer 2, a lattice-like pattern was prevalent on the toes and a homogeneous pattern on the sides of the feet. There was lower inter-observer agreement, with a kappa index of 0.144 .

STUDY LIMITATIONS: The lesions were not biopsied, so clinical-histological correlation could not be performed. The study did not correlate dermoscopic patterns with age.

CONCLUSIONS. As previously reported by other authors, parallel furrows were the most frequently found dermoscopic pattern on palmoplantar skin.
\end{abstract}

Keywords: Dermoscopy; Hispanic americans; Melanoma; Nevus

\section{INTRODUCTION}

Pigmented lesions on acral areas are common and can have different etiologies: skin trauma, post-inflammatory pigmentation, lentigo simplex, melanocytic nevus, and cutaneous melanoma. ${ }^{1-3}$ The differential diagnosis with incipient cutaneous melanoma is often challenging and has increased with this tumor's growing incidence. ${ }^{4}$ From the 1950 s to the 2000s, the total number of mela- noma cases increased by $619 \%$ in the United States. ${ }^{5}$ There are no precise epidemiological data in Latin America. Estimates from the International Agency for Research on Cancer show incidence rates from 0/100,000 inhabitants in Belize to 7.6/100.000 inhabitants in Uruguay. ${ }^{6}$ In Mexico, the Instituto Nacional de Cancerología (INCAN) reported a $500 \%$ increase in incidence in recent years, based on ret-

\footnotetext{
Received 14 November 2016.

Accepted 11 June 2017.

* Work conducted at the Dermatology Outpatient Clinic, Hospital Universitário “Dr. José Eleuterio González", Universidad Autónoma de Nuevo León, Nuevo León, Mexico.

Financial support: None.

Conflict of interest: None.

Department of Introduction to Clinical Practice, Facultad de Medicina, Universidad Autónoma de Nuevo León, Nuevo León, Mexico. Tecnológico de Monterrey, Escuela de Medicina y Ciencias de la Salud, Nuevo León, Mexico.

Dermatology Service, Hospital Universitario “Dr. José Eleuterio González”, Universidad Autónoma de Nuevo León, Nuevo León, Mexico.
}

MAILING AdDREsS:

Roger Adrián González-Ramírez

E-mail: rogergzz@gmail.com 
rospective data from the Histopathological Registry of Malignant Neoplasms, plus an estimated overall incidence of melanoma of $1.01 / 100,000$ inhabitants. ${ }^{7,8}$

Palmoplantar melanocytic nevi are common, thus emphasizing the importance of knowledge of the characteristics that differentiate them from melanoma in the same region. ${ }^{9}$ Dermoscopy (epiluminescence microscopy, surface microscopy) is a non-invasive in vivo test used to study pigmented skin tumors, allowing to increase the diagnostic sensitivity and specificity of cutaneous melanoma. ${ }^{10,11}$

The clinical and dermascopic features of acral pigmented lesions have been obtained from European and Asian populations..$^{2-4,12,13}$ The current study aimed to identify the dermascopic features of melanocytic lesions located on the palmoplantar region in the Latin American population of Mexico. We describe the dermascopic patterns found by two observers with experience in dermoscopy and determined the frequencies and inter-observer agreement.

\section{METHODS}

This was an observational cross-sectional study that included Hispanic patients up to 99 years of age with palmoplantar melanocytic lesions.

Patients were seen at the Dermatology Outpatient Clinic of Hospital Universitário Dr. José Eleuterio González in Monterrey, Mexico from June to August 2014. The study sample excluded patients with acral pigmented lesions with dermoscopic features of acral lentiginous melanoma (ALM), as well as subungual lesions.

The sample also excluded patients with a history of use of immunosuppressants (azathioprine, biological agents, prednisolone, methotrexate, mofetil mycophenolate), current or previous use of alpha-MSH (Melanotan ${ }^{\circledR}$ ), human immunodeficiency virus (HIV) infection, organ transplants, phototherapy, acral skin burns, palmoplantar involvement in psoriasis, pityriasis rubra pilaris, Stevens-Johnson syndrome, erythema multiforme, and bullous diseases.

The clinical and demographic data obtained for the patients included in the study were expressed as percentages: age, sex, skin phototype, personal history of melanoma or non-melanoma skin cancer, presence of dysplastic nevus syndrome, number of melanocytic lesions, site, and dermoscopic pattern (Table 1).

Each pigmented lesion was examined with a DermLite II Pro HR polarized light dermatoscope (3Gen, San Juan Capistrano, CA, USA). A clinical photographic and dermoscopic record was taken with an HP Photosmart R 707 5.1 MP digital camera (Hewlett-Packard Development Company, LP, Palo Alto, CA, USA). The palms were divided anatomically into five areas: thenar, hypothenar, mid-palmar, volar surface of fingers, and lateral surfaces of hands. The soles were divided as follows: plantar arch, volar surface of toes, heel, metatarsal heads, and sides of feet.

The resulting clinical and dermoscopic images were evaluated independently by two dermatologists with experience in dermoscopy (RG and VG), who analyzed and classified the lesions according to the predominant dermoscopic pattern.

The dermoscopic findings were classified in nine different patterns, as described previously in the literature: parallel furrow pattern (PFP), fibrillar, nontypical, lattice-like, homogeneous, globular, reticular, reticulo-globular, and transition. ${ }^{12,13}$ Data were ex-
TABLE 1: Demographic characteristics of the study population
Patients included in the study

Women

Men

Mean age

Skin phototype

I

II

III

IV

321

$223(69.5 \%)$

$98(30.5 \%)$

37.2 years (range: $1-79)$

History

Personal

Non-melanoma skin cancer

$0 \%$

Melanoma

Dysplastic nevus syndrome

Number of lesions

$1-8(2.24)$

Site

Palms

$83.5 \%$

Soles

$16.5 \%$

Side

Right

$50.4 \%$

Left
Family

pressed as means, frequencies, and percentages. The McNemar test was used to compare proportions between observers. Kappa index was calculated as the measure of inter-observer agreement. Statistical significance (alpha value) was set at $5 \%$. Statistical analysis used Microsoft Excel 2013 (Microsoft, Redmond, Washington, USA).

\section{RESULTS}

A total of 4,718 patients with various skin conditions, including those different from the ones included in the study, were evaluated at the Dermatology Outpatient Clinic. Of these patients, $321(6.8 \%)$ had palmoplantar pigmented lesions. From this population, a total of 582 pigmented lesions were evaluated clinically and dermoscopically. Eighty images from 21 patients were excluded due to their low photographic quality. One lesion was excluded from the study because the image presented dermoscopic features of ALM, later confirmed by histopathological examination. The final data resulted in a total of 300 patients with 502 acral melanocytic lesions. Table 1 shows the demographic data.

As for the anatomical location, the majority of the lesions were located on the volar surface of the fingers $(24.3 \%)$, followed by the sides of the hands - Wallace line (WL) (19.9\%); thenar region (14.7\%), hypothenar (12.0\%), mid-palmar (12.5\%); plantar arch $(7.6 \%)$, sides of feet - WL (5.6\%), volar surface of toes $(1.6 \%)$, metatarsal heads (1\%), and heel (0.8\%) (Figure 1$)$. 


\section{Dermoscopic patterns}

The predominant patterns found by observer 1 were: PFP $(62.4 \%)$, homogeneous $(9.2 \%)$, lattice-like $(8.8 \%)$. fibrillar (6.6\%), reticular $(5.8 \%)$, transition (3\%), nontypical $(2 \%)$, globular $(2 \%)$, and reticulo-globular) $(0.2 \%)$. When the lattice-like and fibrillar patterns are included as variants of PFP (model 2), the proportion of lesions classified as PFP increases to $77.8 \%$, followed by the homogeneous $(9.2 \%)$ and reticular $(5.8 \%)$ patterns.

According to observer 2, the predominant patterns were: PFP (40\%), lattice-like (26.1\%), homogeneous (21.7\%), nontypical
$(3.6 \%)$, reticular $(3.0 \%)$, fibrillar $(1.8 \%)$, globular $(1.8 \%)$, reticulo-globular $(1.6 \%)$, and transition $(0.4 \%)$. When lattice-like and fibrillar are included as variants of PFP (model 2), the distribution is as follows: PFP (67.9\%), homogeneous (21.7\%), nontypical (3.6\%), reticular $(3 \%)$, retículo-globular $(1.6 \%)$, and transition $(0.4 \%)$.

For both observers, PFP predominated on the various anatomical sites, but according to observer 2, the lattice-like pattern predominated on the toes and the homogeneous pattern on the sides of the feet (Figure 2).

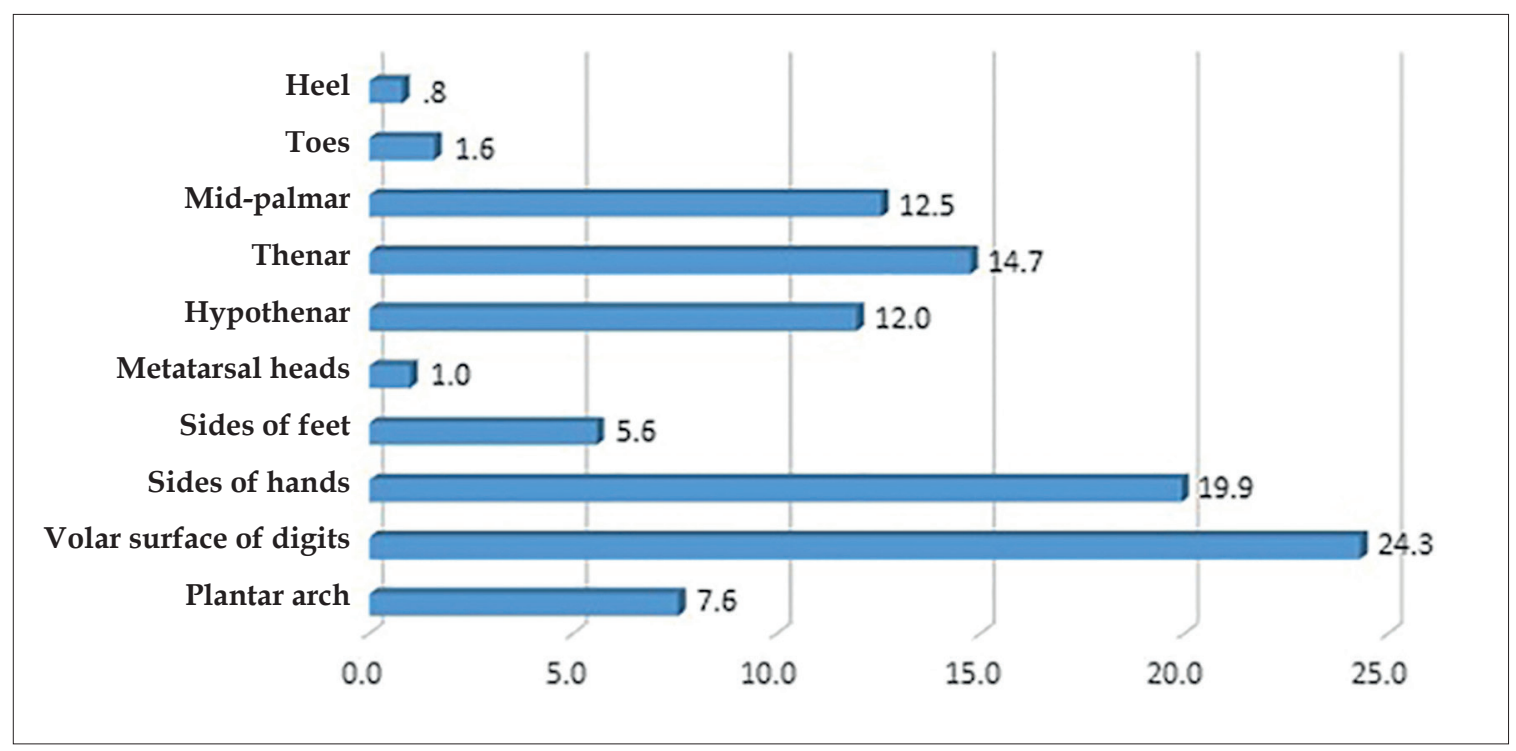

FIGURE 1: Anatomical distribution of lesions

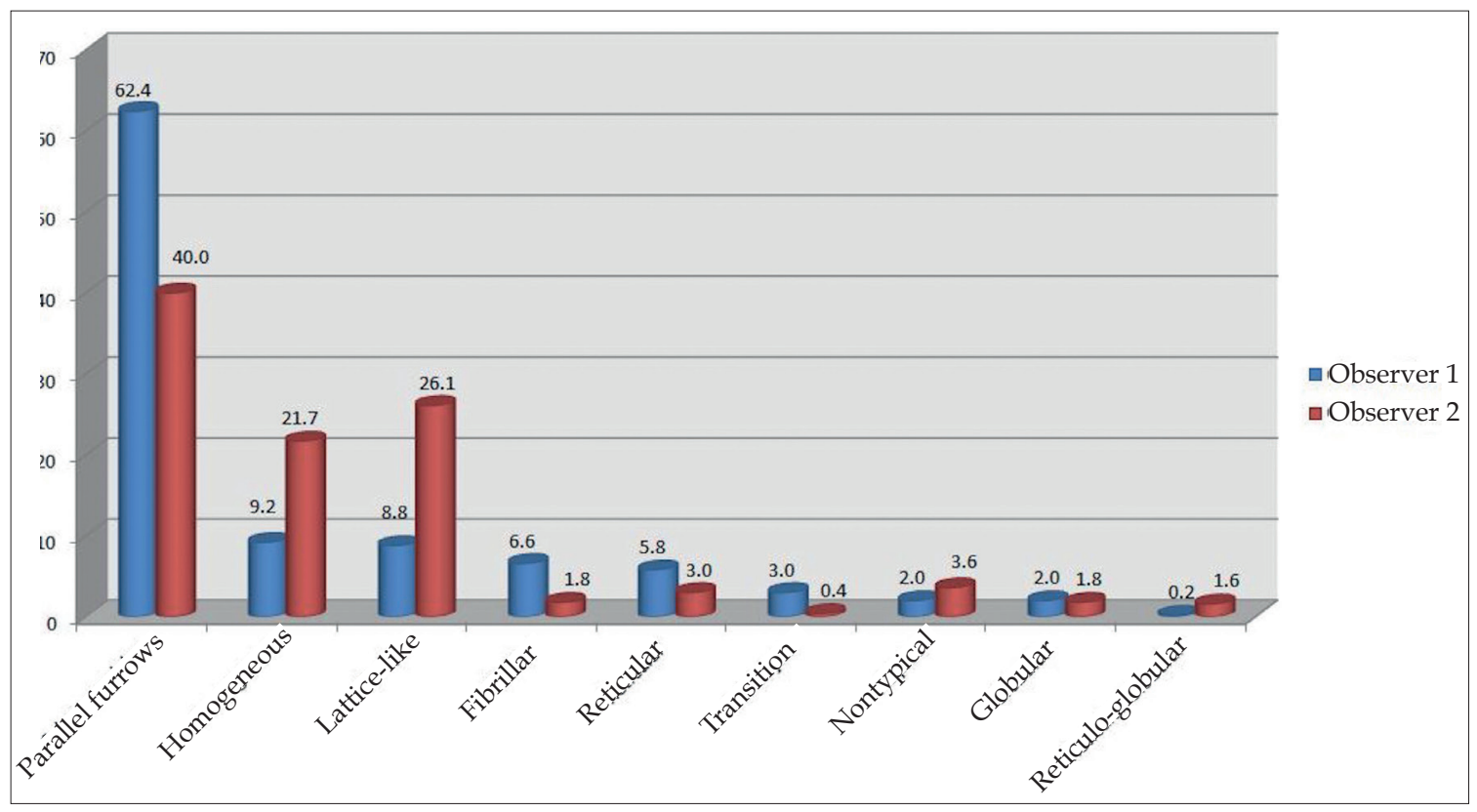

FIGURE 2: Dermoscopic features of acral melanocytic nevi: interobserver agreement 
As mentioned, PFP was the most frequent dermoscopic pattern according to both observers. When the lattice-like and fibrillar patterns were included as variants of PFP, the homogeneous pattern was the second most frequent. The third most common pattern was reticular (5.8\%) for observer 1 and nontypical (3.6\%) for observer 2.

When PFP was subdivided (pure PFP, lattice-like, and fibrillar), there was slight agreement (39.8\%) between the observers, with a kappa index of 0.144; however, when the fibrillar and lattice-like patterns were classified as variants of PFP, the agreement increased to $64.5 \%$, with a kappa of 0.204 . These data are acceptable, according to Landis and Kochs. ${ }^{14}$

When analyzed by anatomical site, the most frequent pattern on the hands according to both observers was PFP, followed by lattice-like and homogeneous. However, observer 1 classified more lesions as PFP (65.4\%) when compared to observer 2 (41.1\%). This difference was statistically significant $(\mathrm{p}=0.017)$. Observer 2 classified more lesions as lattice-like (28.6\%) and homogeneous (19.8\%), compared to observer 1 (9.1\% and $6.9 \%$, respectively), which was also statistically significant ( $\mathrm{p}<0.05 \%$ ).

No statistically significant difference $(p>0.05)$ was seen in the classification of lesions on the soles. PFP was the most frequent pattern, followed by the homogeneous and lattice-like patterns.

\section{DISCUSSION}

Acral lentiginous melanoma (ALM) is the most common variant of melanoma in the non-white population (Asians and African Americans). Radial growth is the predominant component in this type of neoplasm, which may explain why changes in the size of pigmentation are the first sign noticed by patients and physicians. ALM is frequently located on the palms, soles, and nails, most specifically on the nails of the halluces and thumbs..$^{15}$ Incidence is estimated at 0.3 cases per 100,000 inhabitants/year, accounting for an estimated $4.5-7 \%$ of all melanomas. ${ }^{16,17}$ In the black population, ALM accounts for $50-70 \%$ of cases. ${ }^{18}$ In Mexico, $24-31 \%$ of melanomas are acral lentiginous. ${ }^{7}$ A previous study by De la Fuente et al. ${ }^{19}$ reported $23 \%$ prevalence of ALM cases in our outpatient clinic.

One of the main challenges in daily dermatology practice is early diagnosis of cancers on clinical examination, which is difficult even for experienced dermatologists. Dermoscopy increases the diagnostic accuracy of malignant melanocytic lesions, and the different dermoscopic patterns allow the identification of benign lesions, avoiding unnecessary biopsies. ${ }^{2-4,12,13,20-23}$

The aim of this study was to determine the prevalence of palmoplantar pigmented lesions. We also sought to determine whether the palmoplantar patterns described in our population were similar to those in other populations. Finally, we assessed the correlation between the dermoscopic findings of two experienced observers.

For both observers, the predominant dermoscopic pattern was PFP (62.4\% for observer 1 and $40 \%$ for observer 2$)$, similar to the findings in other studies (42-59\%). ${ }^{13,22,24,25}$ For observer 1, the second most frequent pattern was homogeneous $(9.2 \%)$. This pattern has not been reported as the second most frequent one on volar skin. For Ozdemir et al..$^{25}$, the homogeneous pattern was only present in $6.4 \%$ of the cases studied. The third most frequent pattern for observer
1 was lattice-like $(8.8 \%)$. For observer 2 , the second most common pattern was lattice-like, found in $26.1 \%$ of the cases. The frequency of this pattern in our study (26.1\%) was higher than in other studies (13-15\% of cases). ${ }^{13,22,24}$ The homogeneous pattern was the third most frequent. These data are very similar to those of Elwan et al., who detected PFP in $66.1 \%$ of cases, followed by the lattice-like pattern $(8.9 \%)$ and the homogeneous pattern $(7.6 \%) .{ }^{26}$

Inter-observer agreement was low, with a kappa index of 0.144 . The explanation may be that there was a statistically significant difference between the observers in classifying the lesions. For example, observer 1 classified more lesions as PFP (62.5\%), while observer 2 classified more lesions as lattice-like (26.1\%) and homogeneous (21.7\%).

When disaggregating by anatomical sites, the two observers differed in their classification of palmar lesions. Observer 1 found the following frequencies: PFP $65.4 \%$, lattice-like $9.1 \%$, and homogeneous $6.9 \%$. Observer 2 found $41.1 \%$, 28.6\%, and 19,8\%, respectively. The difference was statistically significant. There was no statistically significant difference on the plantar region. There was no statistically significant distribution of dermoscopic patterns within the plantar region. Miyazaki et al. found the majority of lattice-like nevi on the plantar arch. ${ }^{27}$

The low inter-observer agreement may have been due to classification bias, since some nevi can display a combination of two or three patterns in the same lesion. Thus, the classification criterion may have varied according to the observer. ${ }^{21}$ Observer 1 classified more lesions than observer 2 as PFP. This may be explained by the fact that the fibrillar and lattice-like patterns are variants of PFP, which would hinder discrimination between these patterns. Braun et al. observed a low level of agreement in difficult or challenging lesions. $^{28}$

The transition pattern described by Altamura et al..$^{22}$ consists of a combination of a brownish black network in an area of the lesion with parallel furrows or fibrillar pattern in another part of the lesion. It is observed mainly on the sides of the fingers, where the volar skin becomes glabrous. Observer 2 in our study identified these same results, with lesions classified as transition pattern on the sides of the fingers.

One limitation to our study was that no biopsies were performed, thus impeding any clinical-histopathological correlation. Another limitation was that no correlation was performed between the different dermoscopic patterns and age. Minagawa et al. reported a significant correlation between the frequency of dermoscopic patterns and age. ${ }^{29}$ In their study, the fibrillar and "peas-in-a-pod" patterns (described in acral congenital nevi) were significantly prevalent in individuals under 20 years of age. The nontypical pattern was prevalent in the group over 59 years of age. No significant differences were observed in the frequency of PFPs in the different age brackets. ${ }^{29}$

The current study is one of the few that not only identifed the dermoscopic features of palmoplantar lesions, but also studied their prevalence jointly and separately in a large population of patients. We found a prevalence of $6.8 \%$, similar to that reported in other studies, ranging from $6 \%$ to $8 \%{ }^{2,22}$ A recent study published by Madankumar $\mathrm{R}^{30}$, with cases from a highly heterogeneous popu- 
lation in New York and Miami, found a prevalence of 36\%, far higher than previously reported. This may have been due to the inclusion of patients with skin phototypes V and VI, who are more prone to presenting pigmented lesions on the volar skin. ${ }^{30}$

We found a similar prevalence to that reported in populations from Europe and Japan, with predominantly fair-skinned individuals. In our study, $35.4 \%$ of the patients had skin phototypes III and IV, showing that phototype does not influence the frequency of dermoscopic patterns on palmoplantar skin. Our findings are very similar to those reported in the Latin American literature, but we also determined the correlation in dermoscopic patterns between the two observers. ${ }^{21}$
As mentioned previously, ALM is a particular subgroup of melanoma, rare and potentially fatal, which requires specific treatment. ${ }^{31}$ Performing dermoscopy in all patients with pigmented lesions on the palmoplantar region could make all the difference in prognosis.

\section{CONCLUSION}

Our study found very similar numbers to those from previously published studies. The prevalence of palmoplantar lesions in the Mexican population is similar to that reported by other authors. The parallel furrow pattern was also the most common pattern on acral skin. $\square$

\section{REFERENCES}

1. Margolis RJ, Tong AK, Byers HR, Mihm MC Jr. Comparison of acral nevomelanocytic proliferations in Japanese and Whites. J Invest Dermatol. 1989;92:222S-226S.

2. Saida T. Malignant melanoma on the sole: how to detect the early lesions efficiently. Pigment Cell Res. 2000;13:135-9.

3. Saida T. Malignant melanoma in situ on the sole of the foot: its clinical and histopathologic characteristics. Am J Dermatopathol. 1989;11:124-30.

4. Saida T, Yoshida N, Ikegawa S, Ishihara K, Nakajima T. Clinical guidelines for the early detection of plantar malignant melanoma. J Am Acad Dermatol. 1990;23:37-40.

5. Tsao H, Atkins MB, Sober AJ. Management of cutaneous melanoma. N Engl J Med. 2004;351:998-1012.

6. Gutiérrez-Vidrio RM, Cortés-Lozano M. Confrontando al melanoma en el siglo XXI. Med Cutan Iber Lat Am. 2007;35:3-13.

7. Martínez-Saíd $H$, Cuellar-Hubbe M, Barrón Velásquez E, Padilla RA, HerreraGómez A, López Graniel CM, et al. Epidemiology of cutaneous melanoma in Mexico (1982-2002). Eur J Surg Oncol. 2004;30:163.

8. Jacobo R, Pineda B, León G. Melanoma maligno cutáneo. Perfil epidemiológico en México. Gaceta Mex Oncol. 2003;2:17-22.

9. Saida T, Oguchi S, Ishihara Y. In vivo observation of magnified features of pigmented lesions on volar skin using video macroscope. Usefulness of epiluminescence techniques in clinical diagnosis. Arch Dermatol. 1995;131:298-304.

10. Steiner A, Pehamberger $H$, Wolff $K$. In vivo epiluminescence microscopy of pigmented skin lesions, II: diagnosis of small pigmented skin lesions and early detection of malignant melanoma. J Am Acad Dermatol. 1987;17:584-91.

11. Bafounta ML, Beauchet A, Aegerter P, Saiag P. Is dermoscopy (epiluminescence microscopy) useful for the diagnosis of melanoma? Results of a meta-analysis using techniques adapted to the evaluation of diagnostic tests. Arch Dermatol. 2001;137:1343-50.
12. Saida T, Miyazaki A, Oguchi S, Ishihara Y, Yamazaki Y, Murase S, et al. Significance of dermatoscopic patterns in detecting malignant melanoma on acral volar skin. Arch Dermatol. 2004;140:1233-8.

13. Malvehy J, Puig S. Dermatoscopic patterns of benign volar melanocytic lesions in patients with atypical mole syndrome. Arch Dermatol. 2004;140:538-44.

14. Landis JR, Koch GG. The measurement of observer agreement for categorical data. Biometrics. 1977;33:159-74.

15. Argenziano G, Soyer HP, Chimenti S, Talamini R, Corona R, Sera F, et al. Dermoscopy of pigmented skin lesions: results of a consensus meeting via the Internet. J Am Acad Dermatol. 2003;48:679-93.

16. Carli P, de Giorgi V, Chiarugi A, Nardini P, Weinstock MA, Crocetti E, et al. Addition of dermoscopy to conventional naked-eye examination in melanoma screening: a randomized study. J Am Acad Dermatol. 2004;50:683-9.

17. Wolf K, Jonson RA, Surmond D. Fitzpatrick's color atlas and synopsis of clinical dermatology. 5th ed. New York: McGraw-Hill; 2009.

18. Kuchelmeister C, Schaumburg-Lever G, Garbe C. Acral cutaneous melanoma in Caucasians: clinical features, histopathology and prognosis in 112 patients. $\mathrm{Br} \mathrm{J}$ Dermatol. 2000;143:275-80.

19. De la Fuente A, Ocampo-Candiani J. Melanoma Cutáneo. Gac Med Méx 2010;146:126-35

20. Kukita A, Ishihara K. Clinical features and distribution of malignant melanoma and pigmented nevi on the soles of the feet in Japan. J Invest Dermatol. 1989;92:210S-3S.

21. Barquet V, Dufrechou L, Nicoletti S, Acosta MA, Magliano J, Martínez M, et al. Dermoscopic patterns of 158 acral melanocytic nevi in a Latin American population. Actas Dermosifiliogr. 2013;104:586-92. 
22. Altamura D, Altobelli E, Micantonio T, Piccolo D, Fargnoli MC, Peris K. Dermoscopic patterns of acral melanocytic nevi and melanomas in a white population in central Italy. Arch Dermatol. 2006;142:1123-8.

23. Miyazaki A, Saida T, Koga H, Oguchi S, Suzuki T, Tsuchida T. Anatomical and dermoscopic patterns seen in melanocytic nevi on the soles; a retrospective study. J Am Acad Dermatol. 2005;53:230-6.

24. Saida T, Koga H. Dermoscopic patterns of acral melanocytic nevi. Their variations, changes and significance. Arch Dermatol. 2007;143:1423-6.

25. Ozdemir F, Karaarslan IK, Akalin T. Variations in the dermoscopic features of acquired acral melanocytic nevi. Arch Dermatol. 2007;143:1378-84.

26. Elwan NM, Eltatawy RA, Elfar NN, Elsakka OM. Dermoscopic features of acral pigmented lesions in Egyptian patients: a descriptive study. Int J Dermatol. 2016:55:187-92.

27. Miyazaki A, Saida T, Koga H, Oguchi S, Suzuki T, Tsuchida T. Anatomical and histopathological correlates of the dermoscopic patterns seen in melanocytic nevi on the sole: a retrospective study. J Am Acad Dermatol. 2005;53:230-6.
28. Braun RP, Thomas L, Dusza SW, Gaide 0, Menzies S, Dalle S, et al. Dermoscopy of acral melanoma: a multicenter study on behalf of the international dermoscopysociety. Dermatology. 2013;227:373-80.

29. Minagawa A, Koga H, Uhara H, Yokokawa Y, Okuyama R. Age-related prevalence of dermoscopic patterns in acquired melanocytic nevus on acral volar skin. JAMA Dermatol. 2013;149:989-90.

30. Madankumar R, Gumaste PV, Martires K, Schaffer PR, Choudhary S, FaltoAizpurua L, et al. Acral melanocytic lesions in the United States: Prevalence, awareness, and dermoscopic patterns in skin-of-color and non-Hispanic white patients. J Am Acad Dermatol. 2016;74:724-30.e1.

31. Durbec F, Martin L, Derancourt C, Grange F. Melanoma of the hand and foot: epidemiological, prognostic and genetic features. A systematic review. $\mathrm{Br} \mathrm{J}$ Dermatol. 2012;166:727-39.

\section{AUTHORS'CONTRIBUTIONS}

Roger Adrián González-Ramírez

(iD) ORCID 0000-0002-6532-1679

Statistical analysis, Approval of the final version of the manuscript, Design and planning of the study, Preparation and writing of the manuscript, Collecting, analysis and interpretation of data, Effective participation in research orientation, Intellectual participation in propaedeutic and/or therapeutic conduct of studied cases, Critical review of the literature, Critical review of the manuscript

Carolina Guerra-Segovia

D ORCID 0000-0002-6361-3205

Statistical analysis, Preparation and writing of the manuscript, Collecting, analysis and interpretation of data, Effective participation in research orientation, Critical review of the literature

Verónica Garza-Rodríguez

ORCID 0000-0002-5496-3262

Approval of the final version of the manuscript, Design and planning of the study, Collecting, analysis and interpretation of data, Effective participation in research orientation, Critical review of the manuscript

\section{Pamela Garza-Báez}

ORCID 0000-0003-3063-4787

Statistical analysis, Collecting, analysis and interpretation of data, Effective participation in research orientation, Critical review of the literature

Minerva Gómez-Flores

iD ORCID 0000-0002-7183-7065

Approval of the final version of the manuscript, Effective participation in research orientation, Intellectual participation in propaedeutic and/or therapeutic conduct of studied cases, Critical review of the literature, Critical review of the manuscript

Jorge Ocampo-Candiani D ORCID 0000-0002-0213-0031

Approval of the final version of the manuscript, Effective participation in research orientation, Intellectual participation in propaedeutic and/or therapeutic conduct of studied cases, Critical review of the literature, Critical review of the manuscript

How to cite this article: González-Ramírez RA, Guerra-Segovia C, Garza-Rodríguez V, Garza-Báez P, Gómez-Flores M, Ocampo-Candiani J. Dermoscopic features of acral melanocytic nevi in a case series from Mexico. An Bras Dermatol. 2018;93(5):665-70. 